\title{
INNOVATION LEVEL OF SUSTAINABLE PRACTICES ADOPTED IN INDUSTRIAL ENTERPRISES
}

\begin{abstract}
This research aimed to identify the level of innovation of sustainable practices by industrial companies. This is a descriptive study that made use of a questionnaire answered by 50 industrial companies. The results show that environmental practices at full level by $68 \%$ of businesses are monitoring the risks and opportunities for the organization's activities due to climate change; $56 \%$ of companies surveyed are waste separation; followed by the realization of related health and safety training at work in 52\% of cases surveyed; and $48 \%$ monitoring and recording of injuries, the injury rate, the rate of occupational diseases, lost days, absenteeism and number of work-related fatalities for all workers. Among the practices adopted not stand out incineration (burning mass) (80\% of companies surveyed); hiring indigenous and tribal employees $(68 \%)$; composting $(64 \%)$ and use of surface water in the process. Therefore, the study contributed to the disclosure cleaner called production innovations and also pipe end technologies. Some social practices that signal a commitment of the organizations with human resources and the humanization and also economical focused on continuous improvement.
\end{abstract}

Keywords: Sustainability. Sustainable practices. Sustainable innovation.

Simone Sehnem ${ }^{1}$

\footnotetext{
${ }^{1}$ Universidade do Oeste de Santa Catarina - UNOESC, Brasil

E-mail: simone.sehnem@unoesc.edu.br
} 


\section{INTRODUCTION}

The paradigm of sustainability has sensitized managers to adopt environmental practices, social and economic that are innovative. These practices are also called eco-innovations or sustainable innovations when they present production, operation or application of a good, service, production process, organizational structure or management method or business who is new to the company or to the user and that result, through the life-cycle, minimizing environmental risk, pollution and negative impacts of resource use, covering energy consumption compared to conventional alternatives (Kemp \& Pearson, 2008).

The OECD (2009) presents a concept of eco-innovation repeatedly quoted, which mentions that the term is an innovation that results in a reduced environmental impact, whether this effect is intentional or not. The scope of eco-innovation can go beyond the conventional limits of the company to innovate and engage a broader social system, which causes changes in the socio-cultural norms and eco-innovations institutional. As structures are often driven by regulation and legislation, although studies reveal the positive role played by especially clean technologies (Horbach \& Rammer \& Renning, 2014; Horbach, 2008; Frondel et al, 2007).

Despite the diversity of concepts found in the literature for eco-innovation terms, organic practice and innovative sustainable innovation in this study are adopted such terms as synonyms - since there are terms that have high conceptual similarity. The main determinants for the adoption of eco-innovation are associated with specific factors of the company, technological pressure, the pressure of the market and the regulatory framework (Horbach \& Rammer \& Renning, 2014; Horbach 2010). Accordingly, Frondel et al (2007) highlights the effects of regulation may differ with respect to different technological fields. On the one hand, online technologies are activated by specific regulation, cost reduction and environmental management systems are considered most relevant for the release of cleaner technologies.

Thus, this research aims to identify the level of innovation of sustainable practices by industrial companies. The specific objectives are to: a) analyze how innovative are the economic, social and environmental practices adopted by companies surveyed; b) Check what are the motivating and hindering the adoption of eco-innovative practices; c) assess the benefits arising from the adoption of eco-innovative practices; d) To propose alternatives to incorporation of eco-innovative practices in the generating companies surveyed corporate social responsibility, global opportunities and facilitators for internationalization of companies.

The justification for the adoption of sustainable practices in industrial organizations is associated with several benefits that can be glimpsed from that conduct. Stand out cost reduction, improved corporate image, commitment to society and to sustainability. Kamerer (2009) found that the benefits to consumers have a key role in eco-innovations from the moment in which the product brings value to the consumer. The higher the perceived value, the greater the customer's willingness to pay a premium on the price of the product they are purchasing.

The social relevance for the development of this study is associated with longevity of natural resources, maintaining a healthy balance between economic, social and environmental practices adopted by organizations and the pursuit of continuous improvement that is essential to maintain competitive companies. Above all, practice eco-innovative can generate competitive advantage, create market opportunities and allow the employability of many citizens in organizations that stand out by adopting a compromising position with the company.

The structure of the article comprises the section 2 which deals with theoretical aspects depicting the theme sustainable innovation. Section 3 describes the path to operationalize the study. Section 4 presents and analyzes the results and Section 5 presents the conclusions and is followed by item references. 


\section{THEORETICAL FOUNDATION}

The term "eco-innovation" was first used by Fussler and James (1996) in his book Driving Eco-Innovation, published in 1996. It is considered as a new product or process that adds value to the business and the customer, significantly decreasing environmental impacts. Is the production, application or exploitation of property, service, production process, organizational structure or management or business method that is new to the company or user (James, 1997). The results, during its life cycle, are for a reduction of environmental risks, pollution and the negative impacts of resource use, compared with relevant alternatives (Rennings, 1998; Kemp \& Foxon, 2007; Arundel \& Kemp, 2009 ). Andersen (2008) and Foxon and Andersen (2009) conceptualize eco-innovation as innovation that is able to attract green rents in the market, reducing the net environmental impacts, while creating value for organizations. Already Könnölä, CarrilloHermosilla and Gonzalez (2008) conceptualized as a process of technological and/or social systemic change consisting in the invention of an idea and its application in practice to improve environmental performance. Reid and Miedzinski (2008) describe as the creation of new and competitive efforts of products, processes, systems, designed services and procedures to meet human needs and provide better quality of life for all, with minimal use of the life cycle of natural resources and minimum release of toxic substances.

The eco-innovation can also be understood as the production, assimilation or exploitation of a product, production process, services, management or business method that is new to the organization (developed or adopted) and which results, throughout the life cycle generate a reduction of environmental risks, pollution and other negative impacts of resources use (including energy use) compared to relevant alternatives. Relevant alternatives can be the technology used in a business or a common technology to a sector (Kemp \& Pearson, 2008; Coelho, 2015).

Innovation for sustainability can be created in 3 ways: a) by adding components to the conventional system, like the filters to reduce pollution; b) making changes in the sub-systems via improved efficiency in the use of energy, water and materials in manufacturing; c) changing systems via redesign of the production process, product and sales methods to make them more eco-efficient. It cites as an example the structure of a circular production system in which wastes are incorporated into the system again as resources. Covers also open systems, which are generated biodegradable products (or reusable), in which case the resources originally taken from the environment return to nature (Amato Neto, 2015).

Eco innovation does not necessarily involve new knowledge and new technologies and can not originate in the environmental field. Therefore, the spectrum of eco-innovation policy is very broad, its measures require a complex set of indicators including environmental impact. The coordination and stability of jurisdictions and policy instruments are essential, combined with a comprehensive national reference document will facilitate coordination and consistency of improvement, especially if it is based on consistent information. The most efficient policy design takes into account the development of eco-innovations patterns which generate opportunities for the scope of economic cooperation and/or competition (OECD, 2011).

Eco innovations can be categorized by how the companies introduce environmental innovations, namely (Kemp \& Pearson, 2008):

1. Eco Strategic Innovators: active in eco recreational facilities, developing eco innovations to sell to other companies;

2. Eco Strategic Innovators: active in eco recreational facilities, developing eco innovations to sell to other companies; 
3. Innovative eco liabilities: innovations in processes, organizational and products that result in environmental benefits, but where there are no specific strategies to innovate;

4. Not eco innovative: no activities for without pretense or intent innovations with environmental benefits.

Moreover, the paradigm shift to sustainable development permeates the redefinition of innovation for the formation of an ecological market (greener). By providing wide field of knowledge, evolutionary economic approach connects to sustainable development as it positions companies as key players, with features and capabilities that are able to influence change in society, in order to contribute to the economic, environmental development and social (Galvão, 2014). So companies are the main agents of change to create innovation for sustainability and create value for society.

As OECD (2009) eco-innovation is divided into two groups, namely: a) the technology innovations and changes in products or processes b) technological non - innovations in marketing, organizational and institutional.

a) eco-innovations in products and processes: we tend to rely on technological change covering a wide range of tangible goals that can improve environmental conditions and refer technological eco-innovations to reduce or eliminate pollution sources and related to production techniques cleanest

b) eco-innovations in marketing: they include new forms of integration of environmental aspects in communication strategies and sales. Emphasize the company's customer orientation and play a significant role in leveraging the benefits via market research, direct contact, marketing practices (promotion, price, packaging and distribution) that appeal to environmentally conscious customers. The company can achieve improvement or develop ecoefficient products. It also involves new business models.

c) organizational eco-innovations: includes the introduction of new management methods, such as environmental management system, business strategies, centralization, decentralization of environmental responsibility and decision-making, training programs to improve the environmental awareness of employees and the organization's performance. It also includes new forms of relationships with other companies and public organizations and partnerships to develop research and projects;

d) eco-innovation of the institutional structure: includes changes in social norms, the standards of cultural values, beliefs and knowledge, leading to improvements in environmental conditions through the practices and social behavior. Includes structural changes, redefine the roles and relationships through a number of independent entities that imply enforcement of laws, international agreements or voluntary or formal multi-stakeholder arrangements. Ecoinnovative solutions institutional range from water providers, funding for platforms and development of environmental technologies and the establishment of eco-labeling and environmental information systems.

\section{METHODOLOGY}

This survey was sent to 6,472 companies affiliated to the Federation of Industries of the State of Santa Catarina (FIESC). The questionnaire was inserted in google docs and the link sent by e-mail companies. Watched 7 sections, as follows: a) acting branch, b) environmental practices in the production process; c) social practices existing in the company; d) adopted economic practices; d) difficulties to implementation of sustainable practices; e) drivers for the adoption of sustainable practices; f) benefits derived from the adoption of sustainable practices.

There were 7 cases of e-mails that have returned (not valid). Initially (within 10 days) there was return of only 15 completed questionnaires. It was sent new email for the companies 
surveyed giving a new term and the return rate has increased to 50 responses, which corresponds to $0.23 \%$ of the sample - very representative data for a quantitative study. Based on this reality, a research agency was hired to call the companies and seek a more representative sample of completed questionnaires. This part of the study is ongoing. Therefore, this research presents partial data containing the results obtained from the first 50 completed questionnaires.

Data were tabulated in tables and was made a descriptive analysis, to highlight the profile of sustainable practices by Santa Catarina industrial companies. It was later made a profile analysis of sustainable practices in the light of theoretical rules described in the article.

\section{PRESENTATION AND ANALYSIS}

Table 1 describe the field of activity of the companies surveyed.

Table 1: Activity Sector

\begin{tabular}{|c|c|c|c|c|}
\hline Sector & A.F. & R.F & C.F & $\begin{array}{l}\text { Standard } \\
\text { Deviation }\end{array}$ \\
\hline Food products & 13 & $26 \%$ & $26 \%$ & \multirow{14}{*}{4,18} \\
\hline Other sectors & 13 & $26 \%$ & $52 \%$ & \\
\hline Construction & 4 & $8 \%$ & $60 \%$ & \\
\hline Metallurgical & 4 & $8 \%$ & $68 \%$ & \\
\hline Textile & 3 & $6 \%$ & $74 \%$ & \\
\hline Publishing and printing & 2 & $4 \%$ & $78 \%$ & \\
\hline Real estate & 2 & $4 \%$ & $82 \%$ & \\
\hline Paper and cardboard & 2 & $4 \%$ & $86 \%$ & \\
\hline Raw plastic products & 2 & $4 \%$ & $90 \%$ & \\
\hline $\begin{array}{l}\text { Clothing, footwear, fabric } \\
\text { artifact }\end{array}$ & 2 & $4 \%$ & $94 \%$ & \\
\hline Drinks & 1 & $2 \%$ & $96 \%$ & \\
\hline Eraser & 1 & $2 \%$ & $98 \%$ & \\
\hline Mechanics & 1 & $2 \%$ & $100 \%$ & \\
\hline Total & 50 & $100 \%$ & $100 \%$ & \\
\hline
\end{tabular}

* (AF) Absolute Frequency, (RF) Relative Frequency, (CF) Cumulative Frequency Source: Research Data

The survey mapped various industry organizations. Stand out from the food industry, construction and metallurgy.

Table 2: Guidelines implemented in your company

\begin{tabular}{|l|c|c|c|c|}
\hline \multicolumn{1}{|c|}{ Guidelines } & A.F. & R.F. & C.F. & $\begin{array}{c}\text { Standard } \\
\text { Deviation }\end{array}$ \\
\hline 5S & 21 & $36,84 \%$ & $36,84 \%$ & \\
\cline { 1 - 4 } ISO 9.001 & 14 & $24,56 \%$ & $61,40 \%$ & \multirow{2}{*}{$7,47 \%$} \\
\cline { 1 - 4 } None & 14 & $24,56 \%$ & $85,96 \%$ & \\
\cline { 1 - 3 } ISO 14.001 & 6 & $10,53 \%$ & $96,49 \%$ & \\
\cline { 1 - 3 } OSHAS 18.001 & 2 & $3,51 \%$ & $100 \%$ & \\
\hline
\end{tabular}


* Each company can take more than a guideline, so the value exceeded the 50 companies surveyed

** (AF) Absolute Frequency, (RF) Relative Frequency, (CF) Cumulative Frequency

Source: Research Data

Regarding the established guidelines, Table 2 shows that stand out the $5 \mathrm{~S}$ tool and ISO 9,000 . Thus, it is noted that the surveyed companies adopt guidelines to meet the higher standards. Only 6 have an emphasis on practices associated with the environmental management system, possessing the ISO 14001 certification and 2 adopt minimum requirements for best practices in managing occupational health and safety certificate via OSHAS 18001 certification.

Table 3: Stage adoption of environmental practices

\begin{tabular}{|c|c|c|c|c|c|c|c|c|c|c|c|}
\hline \multirow[b]{2}{*}{ Practices } & \multicolumn{2}{|c|}{ Level 1} & \multicolumn{2}{|c|}{ Level 2} & \multicolumn{2}{|c|}{ Level 3} & \multicolumn{2}{|c|}{ Level 4} & \multicolumn{2}{|c|}{ Level 5} & \multirow{2}{*}{$\begin{array}{l}\text { Stand } \\
\text { ard } \\
\text { Devia } \\
\text { tion }\end{array}$} \\
\hline & $\begin{array}{l}\text { A. } \\
\text { F. }\end{array}$ & R.F. & A.F. & $\begin{array}{c}\text { R.F } \\
\text {. }\end{array}$ & A.F. & R.F. & $\begin{array}{c}\text { A.F } \\
\text { • }\end{array}$ & $\begin{array}{l}\text { R. } \\
\text { F. }\end{array}$ & $\begin{array}{l}\text { A. } \\
\text { F. }\end{array}$ & R.F. & \\
\hline Reverse logistic & 5 & $10 \%$ & 8 & $\begin{array}{l}16 \\
\%\end{array}$ & 10 & $20 \%$ & 12 & $\begin{array}{l}24 \\
\%\end{array}$ & 15 & $30 \%$ & 3,81 \\
\hline Cleaner Production & 5 & $10 \%$ & 5 & $\begin{array}{l}10 \\
\%\end{array}$ & 19 & $38 \%$ & 12 & $\begin{array}{l}24 \\
\%\end{array}$ & 9 & $18 \%$ & 5,83 \\
\hline Waste separation & 1 & $2 \%$ & 0 & 0 & 9 & $18 \%$ & 12 & $\begin{array}{l}24 \\
\%\end{array}$ & 28 & $56 \%$ & 11,29 \\
\hline $5 \mathrm{Rs}$ & 3 & $6 \%$ & 8 & $\begin{array}{l}16 \\
\%\end{array}$ & 16 & $32 \%$ & 11 & $\begin{array}{l}22 \\
\%\end{array}$ & 12 & $24 \%$ & 4,85 \\
\hline $\begin{array}{l}\text { Treatment of industrial } \\
\text { effluents }\end{array}$ & 10 & $20 \%$ & 5 & $\begin{array}{l}10 \\
\%\end{array}$ & 4 & $8 \%$ & 12 & $\begin{array}{l}24 \\
\% \\
\end{array}$ & 19 & $38 \%$ & 6,04 \\
\hline Water Recycle & 21 & $42 \%$ & 4 & $8 \%$ & 9 & $18 \%$ & 8 & $\begin{array}{l}16 \\
\%\end{array}$ & 8 & $16 \%$ & 6,44 \\
\hline Water reuse & 20 & $40 \%$ & 6 & $\begin{array}{l}12 \\
\%\end{array}$ & 4 & $4 \%$ & 11 & $\begin{array}{l}22 \\
\%\end{array}$ & 9 & $18 \%$ & 6,20 \\
\hline Pollution control & 11 & $22 \%$ & 4 & $8 \%$ & 12 & $24 \%$ & 13 & $\begin{array}{l}26 \\
\%\end{array}$ & 10 & $20 \%$ & 3,54 \\
\hline Eco-efficiency & 14 & $28 \%$ & 5 & $\begin{array}{l}10 \\
\%\end{array}$ & 10 & $20 \%$ & 17 & $\begin{array}{l}34 \\
\%\end{array}$ & 4 & $8 \%$ & 5,61 \\
\hline Eco-innovation & 8 & $16 \%$ & 6 & $\begin{array}{l}12 \\
\%\end{array}$ & 16 & $32 \%$ & 16 & $\begin{array}{l}32 \\
\%\end{array}$ & 4 & $8 \%$ & 5,66 \\
\hline Biotechnology & 26 & $52 \%$ & 8 & $\begin{array}{l}16 \\
\%\end{array}$ & 8 & $16 \%$ & 6 & $\begin{array}{l}12 \\
\%\end{array}$ & 2 & $4 \%$ & 9,27 \\
\hline $\begin{array}{l}\text { Environmental } \\
\text { management system }\end{array}$ & 10 & $20 \%$ & 11 & $\begin{array}{l}22 \\
\%\end{array}$ & 12 & $24 \%$ & 9 & $\begin{array}{l}18 \\
\%\end{array}$ & 8 & $16 \%$ & 1,58 \\
\hline Clean energy & 12 & $24 \%$ & 11 & $\begin{array}{l}22 \\
\%\end{array}$ & 10 & $20 \%$ & 6 & $\begin{array}{l}12 \\
\%\end{array}$ & 11 & $22 \%$ & 2,35 \\
\hline Eco-design & 16 & $32 \%$ & 10 & $\begin{array}{l}20 \\
\%\end{array}$ & 11 & & 10 & $\begin{array}{l}20 \\
\%\end{array}$ & 3 & $6 \%$ & 4,64 \\
\hline
\end{tabular}




\begin{tabular}{|c|c|c|c|c|c|c|c|c|c|c|c|}
\hline Composting & 32 & $64 \%$ & 7 & $\begin{array}{l}14 \\
\%\end{array}$ & 5 & $10 \%$ & 1 & $2 \%$ & 5 & $10 \%$ & 12,49 \\
\hline $\begin{array}{l}\text { Incineration (mass } \\
\text { burn) }\end{array}$ & 40 & $80 \%$ & 3 & $6 \%$ & 4 & $8 \%$ & 1 & $2 \%$ & 2 & $4 \%$ & 16,81 \\
\hline $\begin{array}{l}\text { Sustainable } \\
\text { consumption }\end{array}$ & 5 & $10 \%$ & 12 & $\begin{array}{l}24 \\
\%\end{array}$ & 20 & $40 \%$ & 10 & $\begin{array}{l}20 \\
\%\end{array}$ & 3 & $6 \%$ & 6,67 \\
\hline $\begin{array}{l}\text { Zero waste (internal } \\
\text { recycling) }\end{array}$ & 11 & $22 \%$ & 13 & $\begin{array}{l}26 \\
\%\end{array}$ & 13 & $26 \%$ & 12 & $\begin{array}{l}24 \\
\%\end{array}$ & 1 & $2 \%$ & 5,10 \\
\hline $\begin{array}{l}\text { Pollution prevention } \\
\text { and control integrated }\end{array}$ & 20 & $40 \%$ & 5 & $\begin{array}{l}10 \\
\%\end{array}$ & 15 & $30 \%$ & 8 & $\begin{array}{l}16 \\
\%\end{array}$ & 2 & $4 \%$ & 7,38 \\
\hline Green chemistry & 27 & $54 \%$ & 6 & $\begin{array}{l}12 \\
\%\end{array}$ & 11 & $22 \%$ & 3 & $6 \%$ & 3 & $6 \%$ & 10,05 \\
\hline $\begin{array}{ll}\text { Use } & \text { of } \\
\text { environmentally } & \\
\text { friendly packaging } & \\
\end{array}$ & 16 & $32 \%$ & 9 & $\begin{array}{l}18 \\
\%\end{array}$ & 10 & $20 \%$ & 11 & $\begin{array}{l}22 \\
\%\end{array}$ & 4 & $8 \%$ & 4,30 \\
\hline Audits to suppliers & 22 & $44 \%$ & 6 & $\begin{array}{l}12 \\
\%\end{array}$ & 8 & $16 \%$ & 4 & $8 \%$ & 10 & $20 \%$ & 7,07 \\
\hline $\begin{array}{l}\text { Audits of internal } \\
\text { processes }\end{array}$ & 13 & $26 \%$ & 4 & $8 \%$ & 8 & $16 \%$ & 8 & $\begin{array}{l}16 \\
\%\end{array}$ & 17 & $34 \%$ & 5,05 \\
\hline $\begin{array}{l}\text { Environmental audits } \\
\text { in production } \\
\text { processes } \\
\text { management and } \\
\text { effluents and waste }\end{array}$ & 26 & $54 \%$ & 0 & 0 & 11 & $22 \%$ & 6 & $\begin{array}{l}12 \\
\%\end{array}$ & 7 & $14 \%$ & 9,77 \\
\hline $\begin{array}{l}\text { Use of surface water in } \\
\text { the processes }\end{array}$ & 31 & $62 \%$ & 3 & $6 \%$ & 3 & $6 \%$ & 4 & $8 \%$ & 9 & $18 \%$ & 12 \\
\hline $\begin{array}{l}\text { Use of groundwater in } \\
\text { the processes }\end{array}$ & 27 & $54 \%$ & 5 & $\begin{array}{l}10 \\
\%\end{array}$ & 6 & $12 \%$ & 7 & $\begin{array}{l}14 \\
\%\end{array}$ & 5 & $10 \%$ & 9,54 \\
\hline $\begin{array}{l}\text { Environmentally sound } \\
\text { management of } \\
\text { hazardous waste }\end{array}$ & 13 & $26 \%$ & 6 & $\begin{array}{l}12 \\
\%\end{array}$ & 10 & $20 \%$ & 13 & $\begin{array}{l}26 \\
\%\end{array}$ & 8 & $16 \%$ & 3,08 \\
\hline $\begin{array}{l}\text { Process technology } \\
\text { that reduces power } \\
\text { consumption }\end{array}$ & 8 & $16 \%$ & 8 & $\begin{array}{l}16 \\
\%\end{array}$ & 20 & $40 \%$ & 10 & $\begin{array}{l}20 \\
\%\end{array}$ & 4 & $8 \%$ & 6 \\
\hline $\begin{array}{l}\text { Process technology } \\
\text { which reduces water } \\
\text { consumption }\end{array}$ & 11 & $22 \%$ & 8 & $\begin{array}{l}16 \\
\%\end{array}$ & 14 & $28 \%$ & 12 & $\begin{array}{l}24 \\
\%\end{array}$ & 5 & $10 \%$ & 3,54 \\
\hline $\begin{array}{l}\text { Process technologies } \\
\text { that reduce the level of } \\
\text { waste }\end{array}$ & 4 & $8 \%$ & 10 & $\begin{array}{l}20 \\
\%\end{array}$ & 12 & $24 \%$ & 15 & $\begin{array}{l}30 \\
\%\end{array}$ & 9 & $18 \%$ & 9,75 \\
\hline $\begin{array}{l}\text { Mitigation of } \\
\text { environmental impacts } \\
\text { generated }\end{array}$ & 20 & $40 \%$ & 6 & $\begin{array}{l}12 \\
\%\end{array}$ & 9 & $18 \%$ & 9 & $\begin{array}{l}18 \\
\%\end{array}$ & 6 & $12 \%$ & 5,79 \\
\hline $\begin{array}{ll}\text { Use coming } & \text { from } \\
\text { renewable fuels } & \end{array}$ & 23 & $46 \%$ & 8 & $\begin{array}{l}16 \\
\%\end{array}$ & 6 & $12 \%$ & 7 & $\begin{array}{l}14 \\
\%\end{array}$ & 6 & $12 \%$ & 7,31 \\
\hline $\begin{array}{lr}\text { Use } & \text { reduction } \\
\text { technologies } & \text { gas } \\
\text { emissions } & \\
\end{array}$ & 20 & $40 \%$ & 6 & $\begin{array}{l}12 \\
\%\end{array}$ & 10 & $20 \%$ & 7 & $\begin{array}{l}14 \\
\%\end{array}$ & 7 & $14 \%$ & 5,79 \\
\hline
\end{tabular}




\begin{tabular}{|l|c|c|c|c|c|c|c|c|c|c|c|}
\hline $\begin{array}{l}\text { Cycle assessment of } \\
\text { product life }\end{array}$ & 17 & $34 \%$ & 7 & $\begin{array}{l}14 \\
\%\end{array}$ & 11 & $22 \%$ & 11 & $\begin{array}{c}22 \\
\%\end{array}$ & 4 & $8 \%$ & 4,90 \\
\hline $\begin{array}{l}\text { Voluntary } \\
\text { environmental } \\
\text { agreements }\end{array}$ & 23 & $46 \%$ & 6 & $\begin{array}{c}12 \\
\%\end{array}$ & 12 & $24 \%$ & 4 & $8 \%$ & 5 & $10 \%$ & 7,91 \\
\hline
\end{tabular}

* (AF) Absolute Frequency, (RF) Relative Frequency, (CF) Cumulative Frequency Source: Research Data

The Table 3 shows that $54 \%$ of the surveyed research have an advanced stage of adoption of reverse logistics practice. Only $10 \%$ of companies surveyed do not adopt this practice and $26 \%$ are in the early stage of any use of reverse logistics (level 2). Cleaner production is a present practice of fullness in $18 \%$ of the surveyed companies and level 4 and over $24 \%$ of companies. This shows that most of the companies surveyed emphasize the continuous application of an integrated preventive environmental strategy to processes, products and services to increase overall efficiency and reduce risks to people and the environment.

The standard deviation of practices ranging from 1.58 to EMS and 16.81 for incineration (burning mass). Another practice that had high variation (12.49) was the compost, then the standard deviation of 12 in item use of surface water in the processes and 11.29 in item waste separation. Among the practices that had the least variation in standard deviation are clean energy (standard deviation 2.35), environmentally sound management of hazardous waste (3.08), process technologies that reduce water consumption $(3,54)$, pollution control $(3.54)$ and reverse logistics (3.81). Among the practices adopted in full form by the researched companies stand out with level 5 waste separation adopted by $56 \%$ of companies, followed by the treatment of industrial effluents with 38\%, audit internal processes $34 \%$ and reverse logistics with $30 \%$. At level 4 there are the eco-efficiency practices with 34\% adoption, with $32 \%$ eco-innovation and process technologies that reduce the level of waste to $30 \%$.

Regarding the practices that are not adopted by the surveyed companies, incineration are (mass burn) in $80 \%$ of the surveyed cases, composting by $64 \%$, use of surface water in the processes $(62 \%)$, green chemistry $(54 \%)$, use of groundwater in the processes $(54 \%)$ and environmental audits in production processes and management of effluents and waste $(54 \%)$. In Level 2 there are the zero waste practices (internal recycling) in $26 \%$ of cases surveyed, sustainable consumption by $24 \%$ of the companies surveyed, clean energy (22\%) and environmental management system $(22 \%)$.

There were some surveyed companies also mentions other practices. Highlights include the recycling of electronic products, construction sprayers filling stations, hospital source material collection, cash in washing stations, power generator use and $100 \%$ of the water used in the company is taken by tanker.

Table 4: Adoption stage of social practices

\begin{tabular}{|c|c|c|c|c|c|c|c|c|c|c|c|}
\hline Practices & \multicolumn{2}{|c|}{ Level 1 } & \multicolumn{2}{|c|}{ Level 2 } & \multicolumn{2}{c|}{ Level 3 } & \multicolumn{2}{|c|}{ Level 4 } & \multicolumn{2}{|c|}{ Level 5 } & Stand \\
& $\begin{array}{c}\text { A. } \\
\text { F. }\end{array}$ & R.F. & A.F. & R.F & A.F. & R.F. & A.F & $\begin{array}{c}\text { R. } \\
\text { - }\end{array}$ & $\begin{array}{c}\text { A. } \\
\text { F. }\end{array}$ & R.F. & $\begin{array}{c}\text { ard } \\
\text { Devia } \\
\text { tion }\end{array}$ \\
\hline Social responsability & 6 & $12 \%$ & 6 & $\begin{array}{c}12 \\
\%\end{array}$ & 13 & $26 \%$ & 18 & $\begin{array}{c}36 \\
\%\end{array}$ & 7 & $14 \%$ & 5,34 \\
\hline $\begin{array}{l}\text { Labor practices based } \\
\text { on universal standards }\end{array}$ & 14 & $28 \%$ & 7 & $\begin{array}{c}14 \\
\%\end{array}$ & 10 & $20 \%$ & 12 & $\begin{array}{c}24 \\
\%\end{array}$ & 7 & $14 \%$ & 3,08 \\
\hline
\end{tabular}




\begin{tabular}{|c|c|c|c|c|c|c|c|c|c|c|c|}
\hline $\begin{array}{l}\text { internationally } \\
\text { recognized practices }\end{array}$ & & & & & & & & & & & \\
\hline $\begin{array}{l}\text { Local employment } \\
\text { discriminating quotas }\end{array}$ & 19 & $38 \%$ & 7 & $\begin{array}{l}14 \\
\%\end{array}$ & 12 & $24 \%$ & 3 & $6 \%$ & 9 & $18 \%$ & 6 \\
\hline $\begin{array}{l}\text { Benefit payments } \\
\text { regularly the } \\
\text { organization of full- } \\
\text { time employees }\end{array}$ & 3 & $6 \%$ & 5 & $\begin{array}{l}10 \\
\%\end{array}$ & 12 & $24 \%$ & 11 & $\begin{array}{l}22 \\
\%\end{array}$ & 19 & $38 \%$ & 6,32 \\
\hline $\begin{array}{l}\text { Monitoring and } \\
\text { recording of injuries, } \\
\text { the injury rate, the rate } \\
\text { of occupational } \\
\text { diseases, lost days, } \\
\text { absenteeism and } \\
\text { number of work- } \\
\text { related fatalities for all } \\
\text { workers (ie employees } \\
\text { and contractors) }\end{array}$ & 7 & $14 \%$ & 5 & $\begin{array}{l}10 \\
\%\end{array}$ & 6 & $12 \%$ & 8 & $\begin{array}{l}16 \\
\%\end{array}$ & 24 & $48 \%$ & 7,91 \\
\hline $\begin{array}{l}\text { Conducting training } \\
\text { regarding health and } \\
\text { safety at work }\end{array}$ & 4 & $8 \%$ & 4 & $8 \%$ & 5 & $10 \%$ & 11 & $\begin{array}{l}22 \\
\%\end{array}$ & 26 & $52 \%$ & 9,41 \\
\hline $\begin{array}{l}\text { Conducting training on } \\
\text { handling of hazardous } \\
\text { waste }\end{array}$ & 11 & $22 \%$ & 6 & $\begin{array}{l}12 \\
\%\end{array}$ & 8 & $16 \%$ & 10 & $\begin{array}{l}20 \\
\%\end{array}$ & 15 & $30 \%$ & 3,39 \\
\hline $\begin{array}{l}\text { Ergonomics training } \\
\text { on performing in the } \\
\text { workplace }\end{array}$ & 8 & $16 \%$ & 7 & $\begin{array}{l}14 \\
\%\end{array}$ & 8 & $16 \%$ & 9 & $\begin{array}{l}18 \\
\%\end{array}$ & 18 & $36 \%$ & 4,53 \\
\hline $\begin{array}{l}\text { Conducting training on } \\
\text { accident prevention in } \\
\text { the workplace }\end{array}$ & 3 & $6 \%$ & 6 & $\begin{array}{l}12 \\
\%\end{array}$ & 8 & $16 \%$ & 10 & $\begin{array}{l}20 \\
\%\end{array}$ & 23 & $46 \%$ & 7,71 \\
\hline $\begin{array}{l}\text { Conducting training on } \\
\text { aspects of human rights } \\
\text { relevant to the } \\
\text { organization's } \\
\text { operations }\end{array}$ & 14 & $28 \%$ & 6 & $\begin{array}{l}12 \\
\%\end{array}$ & 12 & $24 \%$ & 10 & $\begin{array}{l}20 \\
\%\end{array}$ & 8 & $16 \%$ & 3,16 \\
\hline $\begin{array}{l}\text { Hiring indigenous and } \\
\text { tribal employees }\end{array}$ & 34 & $68 \%$ & 5 & $\begin{array}{l}10 \\
\%\end{array}$ & 4 & $8 \%$ & 3 & $6 \%$ & 4 & $8 \%$ & 13,44 \\
\hline $\begin{array}{l}\text { Report the formal } \\
\text { procedures for } \\
\text { complaints and claims } \\
\text { by local communities }\end{array}$ & 17 & $34 \%$ & 10 & $\begin{array}{l}20 \\
\%\end{array}$ & 10 & $20 \%$ & 6 & $\begin{array}{l}12 \\
\%\end{array}$ & 7 & $14 \%$ & 4,30 \\
\hline $\begin{array}{l}\text { Report the significant } \\
\text { risks related to } \\
\text { corruption identified } \\
\text { based on risk } \\
\text { assessments }\end{array}$ & 19 & $38 \%$ & 9 & $\begin{array}{l}18 \\
\%\end{array}$ & 7 & $14 \%$ & 9 & $\begin{array}{l}18 \\
\%\end{array}$ & 6 & $12 \%$ & 5,20 \\
\hline $\begin{array}{lr}\text { Report } & \text { the } \\
\text { anticorruption } & \text { policies } \\
\end{array}$ & 17 & $34 \%$ & 6 & $\begin{array}{l}12 \\
\%\end{array}$ & 7 & $14 \%$ & 13 & $\begin{array}{l}26 \\
\% \\
\end{array}$ & 7 & $14 \%$ & 4,80 \\
\hline
\end{tabular}




\begin{tabular}{|c|c|c|c|c|c|c|c|c|c|c|c|}
\hline $\begin{array}{l}\text { and procedures } \\
\text { adopted by the } \\
\text { organization }\end{array}$ & & & & & & & & & & & \\
\hline $\begin{array}{l}\text { Monitoring the number } \\
\text { of complaints and } \\
\text { claims of customers } \\
\text { and suppliers }\end{array}$ & 7 & $14 \%$ & 3 & $6 \%$ & 9 & $18 \%$ & 9 & $\begin{array}{l}18 \\
\%\end{array}$ & 22 & $44 \%$ & 7,14 \\
\hline $\begin{array}{l}\text { Note the ergonomic } \\
\text { aspects in the processes }\end{array}$ & 10 & $20 \%$ & 5 & $\begin{array}{l}10 \\
\%\end{array}$ & 7 & $14 \%$ & 15 & $\begin{array}{l}30 \\
\%\end{array}$ & 13 & $26 \%$ & 4,12 \\
\hline $\begin{array}{lr}\text { Communicate } & \text { to } \\
\text { stakeholders } & \text { the } \\
\text { sustainable } & \\
\text { performance } & \text { via } \\
\text { specific } & \text { reports } \\
\text { (sustainability report } \\
\text { and social audit) }\end{array}$ & 22 & $44 \%$ & 5 & $\begin{array}{l}10 \\
\%\end{array}$ & 3 & $6 \%$ & 10 & $\begin{array}{l}20 \\
\%\end{array}$ & 10 & $20 \%$ & 7,38 \\
\hline Green marketing & 15 & $30 \%$ & 11 & $\begin{array}{l}22 \\
\%\end{array}$ & 10 & $20 \%$ & 10 & $\begin{array}{l}20 \\
\%\end{array}$ & 4 & $8 \%$ & 3,94 \\
\hline $\begin{array}{l}\text { Report principles and } \\
\text { ethical values of the } \\
\text { company, either in } \\
\text { internal processes and } \\
\text { in negotiations with } \\
\text { stakeholders } \\
\text { (customers, suppliers, } \\
\text { society and } \\
\text { shareholders) }\end{array}$ & 8 & $16 \%$ & 6 & $\begin{array}{l}12 \\
\%\end{array}$ & 8 & $16 \%$ & 13 & $\begin{array}{l}26 \\
\%\end{array}$ & 15 & $30 \%$ & 3,81 \\
\hline
\end{tabular}

* (AF) Absolute Frequency, (RF) Relative Frequency, (CF) Cumulative Frequency

Source: Research Data

The Table 4 shows that the adoption stage of social practices is at full level (level 5) for the practical realization of related health and safety training at work in 52\% of companies surveyed. Monitoring and recording of injuries, the injury rate, the rate of occupational diseases, lost days, absenteeism and number of work-related fatalities for all workers (ie employees and contractors) is practical adopted by $48 \%$ of companies surveyed. And conducting training on accident prevention in the workplace by $46 \%$ of the cases. And still monitoring the number of complain and complaints of customers and suppliers in $44 \%$ of companies. At level 4 we highlight the social responsibility practices (36\% of companies), observation of ergonomic aspects in the processes (30\%); communicate the anticorruption policies and procedures adopted by the organization (26\%) and communicating principles and ethical values of the company, either in internal processes and in negotiations with stakeholders (customers, suppliers, society and shareholders) (26\%).

Regarding the social practices that not adopted highlights are hiring indigenous and tribal employees (68\%), communicate to stakeholders the sustainable performance via specific reports (sustainability report and social audit) in $44 \%$ of companies; report significant risks related to corruption identified based on risk assessments (38\%) and local employment discriminating quotas $(38 \%)$. 
The standard deviation varies from 3.08 to labor practices based on universal standards internationally recognized practices to 13.44 for hiring indigenous and tribal employees.

Table 5: Stage adoption of economic practices

\begin{tabular}{|c|c|c|c|c|c|c|c|c|c|c|c|}
\hline \multirow[b]{2}{*}{ Practices } & \multicolumn{2}{|c|}{ Level 1} & \multicolumn{2}{|c|}{ Level 2} & \multicolumn{2}{|c|}{ Level 3} & \multicolumn{2}{|c|}{ Level 4} & \multicolumn{2}{|c|}{ Level 5} & \multirow{2}{*}{$\begin{array}{l}\text { Stand } \\
\text { ard } \\
\text { Deviat } \\
\text { ion }\end{array}$} \\
\hline & $\begin{array}{l}\text { A. } \\
\text { F. }\end{array}$ & R.F. & A.F. & $\begin{array}{c}\text { R.F } \\
\text {. }\end{array}$ & A.F. & R.F. & $\begin{array}{c}\text { A.F } \\
.\end{array}$ & $\begin{array}{l}\text { R. } \\
\text { F. }\end{array}$ & $\begin{array}{l}\text { A. } \\
\text { F. }\end{array}$ & $\begin{array}{c}\text { R.F } \\
\text {. }\end{array}$ & \\
\hline $\begin{array}{l}\text { Cost of monitoring per } \\
\text { unit produced }\end{array}$ & 5 & $10 \%$ & 3 & $6 \%$ & 10 & $20 \%$ & 9 & $\begin{array}{l}18 \\
\%\end{array}$ & 23 & $\begin{array}{l}46 \\
\%\end{array}$ & 7,81 \\
\hline $\begin{array}{lr}\text { Monitoring rework } \\
\text { index and reprocessing }\end{array}$ & 6 & $12 \%$ & 4 & $8 \%$ & 10 & $20 \%$ & 12 & $\begin{array}{l}24 \\
\%\end{array}$ & 18 & $\begin{array}{l}36 \\
\%\end{array}$ & 5,47 \\
\hline $\begin{array}{l}\text { Monitoring the loss } \\
\text { ratio in the process in } \\
\text { real }\end{array}$ & 5 & $10 \%$ & 5 & $\begin{array}{l}10 \\
\%\end{array}$ & 6 & $12 \%$ & 15 & $\begin{array}{l}30 \\
\%\end{array}$ & 19 & $\begin{array}{l}68 \\
\%\end{array}$ & 6,56 \\
\hline $\begin{array}{l}\text { Monitoring of risks } \\
\text { and opportunities for } \\
\text { the organization's } \\
\text { activities due to } \\
\text { climate change }\end{array}$ & 19 & $38 \%$ & 6 & $\begin{array}{l}12 \\
\%\end{array}$ & 8 & $16 \%$ & 9 & $\begin{array}{l}18 \\
\%\end{array}$ & 8 & $\begin{array}{l}16 \\
\%\end{array}$ & 5,15 \\
\hline $\begin{array}{l}\text { Identifies the } \\
\text { significant indirect } \\
\text { economic impacts, } \\
\text { both positive and } \\
\text { negative }\end{array}$ & 6 & $12 \%$ & 7 & $\begin{array}{l}14 \\
\%\end{array}$ & 15 & $30 \%$ & 13 & $\begin{array}{l}26 \\
\%\end{array}$ & 9 & $\begin{array}{l}18 \\
\%\end{array}$ & 3,87 \\
\hline $\begin{array}{lr}\text { Prioritization } & \text { of } \\
\text { spending on local } \\
\text { suppliers }\end{array}$ & 7 & $14 \%$ & 15 & $\begin{array}{l}30 \\
\%\end{array}$ & 9 & $18 \%$ & 11 & $\begin{array}{l}22 \\
\%\end{array}$ & 8 & $\begin{array}{l}16 \\
\%\end{array}$ & 3,16 \\
\hline
\end{tabular}

* (AF) Absolute Frequency, (RF) Relative Frequency, (CF) Cumulative Frequency Source: Research Data

According to Table 5, 68\% of the surveyed companies adopt in full stage monitoring the loss ratio in the process in real; 46\% monitoring of the cost per unit of output and 36\% monitoring of rework and rework index. As non-adopted practices include the monitoring of risks and opportunities for the organization's activities due to climate change (38\% of companies) and prioritization of spending on local suppliers (12\%).

The standard deviation was 3.16 for prioritization of spending on local suppliers and 7.81 for monitoring the cost per unit produced.

Table 6: Major difficulties to implement sustainable practices in your company

\begin{tabular}{|l|c|c|c|c|}
\hline \multicolumn{1}{|c|}{ Practices } & A.F. & R.F. & C.F. & Standard Deviation \\
\hline $\begin{array}{l}\text { The need to invest in capital (new } \\
\text { machines and equipment) }\end{array}$ & 19 & $38 \%$ & $38 \%$ & \\
\cline { 1 - 3 } Corporate culture & 9 & $18 \%$ & $56 \%$ & \\
\cline { 1 - 3 } Measuring difficulty & 7 & $14 \%$ & $70 \%$ & \\
\hline
\end{tabular}




\begin{tabular}{|l|c|c|c|c|}
\hline Do not know the practices & 5 & $10 \%$ & $80 \%$ & \multirow{2}{*}{5,92} \\
\hline Others & 5 & $10 \%$ & $90 \%$ & \\
\cline { 1 - 3 } $\begin{array}{l}\text { Lack of top management commitment } \\
\text { to implement sustainable actions }\end{array}$ & 4 & $8 \%$ & $98 \%$ & \\
\hline The monitoring of suppliers & 1 & $2 \%$ & $100 \%$ & \\
\cline { 1 - 3 } Risk management & 0 & 0 & $100 \%$ & \\
\hline Total & 50 & $100 \%$ & $100 \%$ & \\
\hline
\end{tabular}

* Podia assinalar mais do que uma alternativa

** (AF) Absolute Frequency, (RF) Relative Frequency, (CF) Cumulative Frequency

Fonte: dados da pesquisa

The Table 6 shows that the need to invest in capital is the biggest impediment to the adoption of sustainable practices; followed by corporate culture and measurement of difficulty. Other difficulties highlighted by respondents were: the size of the company, nationwide, hinders any new action; legislation; lack of investment; public policy bureaucracy; lack of knowledge and commitment to implement sustainable actions. It is not cultural in our country worry about the measures analyzed and when there are charges, there is an immense difficulty in measuring the actions to be taken. It is therefore necessary to resort to hiring of expert advice. When I say that the problem is cultural, I want to indicate that a person who works in any administrative sector of the production, often with higher education, do not know the practice, even the simplest of recycling and waste separation. When more implement a high level of action on a system. The first difficulty I find is precisely explain the importance of separation and proper disposal of waste. I see it as a social problem of lack of education. Deploy awareness of sustainable practices in all the company's employees; company without financial resources and implement awareness.

Table 7: Main motivation to adopt sustainable practices

\begin{tabular}{|c|c|c|c|c|}
\hline Practices & A.F. & R.F & C.F. & Standard Deviation \\
\hline $\begin{array}{l}\text { Awareness of managers of their need } \\
\text { and importance }\end{array}$ & 18 & $36 \%$ & $36 \%$ & \multirow{13}{*}{4,51} \\
\hline Corporate culture & 5 & $10 \%$ & $46 \%$ & \\
\hline $\begin{array}{l}\text { External pressures } \\
\text { shareholders, NGOs, } \\
\text { community at large) }\end{array}$ & 4 & $8 \%$ & $54 \%$ & \\
\hline Cost reduction & 4 & $8 \%$ & $62 \%$ & \\
\hline Impact on corporate image & 4 & $8 \%$ & $70 \%$ & \\
\hline $\begin{array}{l}\text { The desire to be respected by the } \\
\text { community }\end{array}$ & 3 & $6 \%$ & $76 \%$ & \\
\hline Risk management & 3 & $6 \%$ & $82 \%$ & \\
\hline Increased profit & 2 & $4 \%$ & $86 \%$ & \\
\hline Increased operational efficiency & 2 & $4 \%$ & $90 \%$ & \\
\hline Concern about the brand & 2 & $4 \%$ & $94 \%$ & \\
\hline Internal pressures (of employees) & 1 & $2 \%$ & $96 \%$ & \\
\hline Regulatory concerns & 1 & $2 \%$ & $98 \%$ & \\
\hline Others & 1 & $2 \%$ & $100 \%$ & \\
\hline Total & 50 & $100 \%$ & $100 \%$ & \\
\hline
\end{tabular}


* It may be noted more than one alternative

** (AF) Absolute Frequency, (RF) Relative Frequency, (CF) Cumulative Frequency

Source: Research Data

The Table 6 as the main reasons for the adoption of environmental practices in business are the awareness of managers of their necessity and importance, corporate culture; external pressures (customers, shareholders, NGOs, government, community at large); reducing costs and impact on corporate image. Other reasons highlighted by respondents are: the company's area of operation; we are living in the age anthropocentric, we must take action independently of external collections and maintenance of the environment.

Table 7: Benefits seen the adoption of sustainable practices

\begin{tabular}{|c|c|c|c|c|}
\hline Practices & A.F.* & R.F.* & C.F.* & $\begin{array}{l}\text { Standard } \\
\text { Deviation }\end{array}$ \\
\hline Best picture & 14 & $28 \%$ & $28 \%$ & \multirow{9}{*}{4,53} \\
\hline Quality improvement & 10 & $20 \%$ & $48 \%$ & \\
\hline Improved management & 7 & $14 \%$ & $62 \%$ & \\
\hline Higher profitability & 7 & $14 \%$ & $76 \%$ & \\
\hline Growth & 6 & $12 \%$ & $88 \%$ & \\
\hline Low cost & 2 & $4 \%$ & $92 \%$ & \\
\hline $\begin{array}{lll}\begin{array}{l}\text { Research } \\
\text { improvement }\end{array} & \text { and development } \\
\end{array}$ & 2 & $4 \%$ & $96 \%$ & \\
\hline Others & 2 & $4 \%$ & $100 \%$ & \\
\hline Pioneering & 0 & 0 & 0 & \\
\hline Total & 50 & $100 \%$ & $100 \%$ & \\
\hline
\end{tabular}

* (AF) Absolute Frequency, (RF) Relative Frequency, (CF) Cumulative Frequency Source: Research Data

As shown in Table 7, are considered the main benefits of adopting environmental practices to improve the image, quality, management and profitability. Other reasons given are: internal and external quality, great acceptance to the citizens, improving the understanding of the human aspect of the production system and even about the occupation of the planet; improving the working environment and quality of life of all people involved in the production process; improvement of management; preservation awareness.

Interestingly environmental practices are not adopted in business surveys also had the highest standard deviation, that is, greater variability in values. This behavior was not repeated in social practices where only two practices not taken also had high rates on standard deviation. And as regards the economic practices is evident that the practices that may be considered proactive and eco-innovative are not adopted in most of the companies surveyed and had higher levels of variability in the standard deviation indicator.

Horbach, Rammer and Renning (2014) mention that environmental innovations and economically benign are not carried out in many organizations because of incomplete information and organizational and coordination problems. Companies are unable to recognize the potential of eco-innovation of cost reduction. Kanna et al (2009) reiterate how important managers have a broader view of environmental management systems, to promote the engagement of the management summit, empowering employees at all levels and techniques 
such as process mapping, analysis the generating and environmental accounting causes. Such conduct leave companies aware of the inefficiencies that were not previously recognized and to discover new ways to increase efficiency and reduce pollution control costs.

Based on this scenario mapped, this research proposes alternatives for incorporating ecoinnovative practices in the generating companies surveyed corporate social responsibility and global opportunities.

Figure 1: Recommendations for organizations in the light of eco-innovation types recommended by the OECD

\begin{tabular}{|l|l|}
\hline Eco-innovations & \multicolumn{1}{|c|}{ Recommendations for Organizations } \\
\hline Technological & - Cleaner Production technologies Adoption \\
& - Incorporation of eco-efficiency practices \\
- Environmental Management System Implementation & - Development of Product Life Cycle Analysis \\
& - Control technology adoption and prevention technologies \\
- Preference in the purchase of green products & - Monitoring of environmental criteria for selecting suppliers \\
& - Adopt recycle water \\
- Adopt always possible local purchases & - Investing in R \& D in ecodesing, use of ecological raw materials \\
and lean production processes and sustainable \\
- Embedding processes to reduce the use of natural resources in \\
operations \\
- Selection of clean transportation methods \\
- Use containers and reusable or recyclable containers \\
- Use of environmentally friendly materials for primary packaging \\
- Retrieval system Adoption of materials and recycling \\
- Separation, preparation and responsible waste disposal \\
- Replacement of hazardous materials and pollutants \\
- Innovating in storage practices, distribution and marketing of \\
products with a view to promoting social and environmental \\
sustainability \\
- Reducing air emissions, wastewater and solid waste \\
- Reducing the consumption of water and energy in the process of \\
production;
\end{tabular}




\begin{tabular}{|l|l|}
\hline - Selection of clean transportation methods \\
- Use containers and reusable or recyclable containers \\
- Use of environmentally friendly materials for primary packaging \\
- Retrieval system Adoption of materials and recycling \\
- Separation, preparation and responsible waste disposal \\
- Replacement of hazardous materials and pollutants \\
- Innovating in storage practices, distribution and marketing of \\
products with a view to Promoting social and environmental \\
sustainability \\
- Reducing air emissions, wastewater and solid waste \\
- Reducing the consumption of water and energy in the process of \\
production; \\
- Investing in Corporate Social Responsibility (standards, reports \\
and voluntary codes) \\
- Product development, green brand, ecological packaging, sales and \\
sustainable logistics \\
- Adopting green marketing \\
- Preparation of periodic environmental reports \\
- Internal documentation of not environmental and social \\
compliance \\
- Sponsorship of environmental and social events \\
- Regular Voluntary information on environmental management for \\
clients and institutions \\
- Adoption of environmental emergency plans \\
- Have clear goals and make long-term environmental planning \\
- Measure and evaluate the economic, social and environmental \\
performance \\
- Set explicitly the current environmental policy in the company \\
- Total dedication of the employees to perform the environmental \\
management and social \\
- Training programs for Adoption in social and environmental \\
questions for all employees \\
- Dissemination of ethical conduct and transparency within the \\
organization \\
- Adoption of social norms such as SA 8000 and OSHAS 18001 \\
- Adoption of environmental standards such as ISO 14001 \\
- Interaction research institutes, technology centers, universities to \\
generate innovations in products and processes; \\
\hline
\end{tabular}

Source: The author (2015)

\section{FINAL CONSIDERATIONS}

This research aimed to identify the level of innovation of sustainable practices by industrial companies. The results show that the practices that are adopted in full level in various companies are separating waste, treatment of industrial effluents, audit of internal processes, reverse logistics; 5rs; clean energy, conducting related health and safety training at work, monitoring and recording of injuries, the injury rate, the rate of occupational diseases, lost days, absenteeism and number of work-related fatalities to total workers (ie employees and 
contractors); training on accident prevention in the workplace; monitoring the number of complaints and claims of customers and suppliers; granting benefits to regular full-time employees of the organization; training sessions on ergonomics in the workplace; monitoring the loss ratio in the process in real; monitoring the cost per unit of output; monitoring of rework and rework index; identifies the significant indirect economic impacts, both positive and negative; monitoring of risks and opportunities for the organization's activities due to climate change; and prioritization of spending on local suppliers.

The practices cited as not adopted by various companies surveyed are: incineration (burning mass); composting; use of surface water in the processes; green chemistry; use of groundwater in the processes; environmental audits in production processes and management of effluents and waste; hiring indigenous and tribal employees; communicate to stakeholders the sustainable performance via specific reports (sustainability report and social audit); report significant risks related to corruption identified on the basis of risk assessments; employees of discriminating hiring quotas; report the formal procedures for complaints and claims by local communities; communicate the anticorruption policies and procedures adopted by the organization; monitoring of risks and opportunities for the organization's activities due to climate change; prioritization of spending on local suppliers; monitoring of rework and rework index; identifies the significant indirect economic impacts, both positive and negative; monitoring the loss ratio in the process in real; monitoring the cost per unit produced.

It is important to remember that eco-innovation differs from the classical concept of innovation by relating to the reduction of environmental burdens, ie, an innovation that consists of changes and improvements in environmental performance within a greening context of products, processes, strategies business, markets, technology and innovation systems. Soon, eco-innovation has direct contribution to reducing the environmental impact of products and processes.

Therefore, research contributions are associated with the highlighted cleaner called production innovations and also pipe end technologies. Some social practices that signal a commitment of the organizations with human resources and the humanization and also economical focused on continuous improvement. Above all, it is noticeable that each company is a locus which is a progressive accumulation of resources and technological expertise and has very specific and idiosyncratic elements of the company and the country where it was conceived, developed and improved. And eco-innovations are the development of an organization's efforts to maintain, improve and renew the social and environmental quality, the benefits are extended to the whole society, represented by their business processes, including products and services. Derivatives benefits for society are the reduction of environmental pollution, limited use of natural resources, accountability to stakeholders and contributions to social problems.

As a recommendation for future studies suggests to analyze the multiple areas of impact for both eco-innovations related to processes as with products, highlighting the importance of these impacts on the total contribution of the company in relation to the reduction of environmental externalities. are sources of competitiveness, enable occupy market opportunities. The identification of specific determinants of eco-innovation by environmental impact area can help them formulate more detailed policy recommendations that are best suited to different market segments 


\section{REFERÊNCIAS}

Amato Neto, J. (2015). A era do eco-business: criando negócios sustentáveis. Baureri, SP: Manole.

Andersen, M. M. (2008). Eco-innovation - towards a taxonomy and a theory. In: DRUID Conference - Entrepreneurship and Innovation - Organizations, Institutions, Systems and Regions, 25, jun. 2008, Copenhagen.

Arundel A.; Kemp, R. (2009). Measuring eco-innovation. UNU-MERIT Working Paper Series, 2009. Disponível em: < http://www.merit.unu.edu/publications/wppdf/2009/wp2009017.pdf >. Acesso em: 10 out. 2015.

Coelho, M. A. (2015). Ecoinovação em uma pequena empresa de reciclagens da cidade de Manaus Revista de Administração e Inovação, São Paulo, v.12, n.1, p.121-147, jan./mar. 2015

Foxon, T.; Andersen, M. M. (2009). The greening of innovation systems for eco-innovation - towards an evolutionary climate mitigation policy. In: DRUID Summer Conference Innovation, Strategy and Knowledge, jun. 2009, Copenhagen

Frondel, M.; Horbach, J; Rennings, K. (2007). End-of-pipe or cleaner production? An empirical comparison of environmental innovation decisions across OECD countries. Business strategy and the environmental. vol. 16. n.8. 571-584.

Fussler, C.; James, P. (1996). Driving Eco-innovation: a breakthrough discipline for innovation and sustainability. London: Pitman Publishing, 1996.

Galvão, H.M. (2014). Influência da gestão sócio-ambiental no desempenho da eco-inovação empresarial. Tese de doutorado. 237p. Programa de Pós-Graduação em Administração FEA/USP. São Paulo.

Horbach, J (2008). Determinants of environmental innovation: new evidences from German Panel data sources. Research Policy, vol.37. 163-173.

Horbach, J (2010). The impact of innovation activities on employment in the environmental sector - empirical results for Germany at the firm level. In: Jahrbücher für Nationalökonomie und Statistik/ Journal of Economics and Statistics, vol.230/4, 403-419.

Horbach, J.; Rammer, C; Rennings, K. (2014). Determinantes da ecoinovação por tipo de impacto ambiental: o papel da pressão regulatória, da alavancagem tecnológica e do fator de mercado. IN: Arruda, C; Carvalho, F. (2014). Inovações ambientais: políticas públicas, tecnologias e oportunidades de negócios. Rio de Janeiro: Elsevier.

James, P. (1997). The Sustainability Circle: a new tool for product development and design. Journal of Sustainable Product Design, n. 2, p. 52-57.

Kammerer, D (2009). The effects of customer benefit and regulation on environmental product innovation. Empirical evidences from appliance manufacturers in Germany. Ecological Economics, v.68, 2285-2295. 
Kemp, R.; Pearson, P. (2008) Final report MEI project about measuring eco-innovation. UM-MERIT. Maastricht, www.merit.unu.edulMEI.

Kemp, R.; Foxon, T. J. (2007). Tipology of Eco-Inovation. In: MEI project: measuring EcoInovation. European Commission, ago. 2007. Disponível em: . Acesso em: 15 out. 2015.

Könnölä, T.; Carrillo-Hermosilla, J.; Gonzalez, P. del R. (2008). Dashboard of ecoinnovation. In: DIME International Conference - Innovation, sustainability and policy, sep. 2008, University Montesquieu Bordeaux IV, France.

OECD - Organisation for Economic Co-Operation and Development. Eco-innovation in industry: enabling green growth. 2009. Disponível em: http://www.imamidejo.si/resources/files/eco_innovation_oecd.pdf. Acesso em 18 out.2015.

OECD - Organisation for Economic Co-Operation and Development.. Manual de Oslo: diretrizes para coleta e interpretação de dados sobre inovação. 3. ed. Trad. FINEP. Rio de Janeiro: OECD; Eurostat; FINEP, 2005. Disponível em: Acesso em: 05 out 2015

Reid, A.; Miedzinski, M. (2008). Eco-Innovation, Final Report for Sectoral Innovation Watch. Brussels: Technopolis Group, 2008. Disponível em: < http://www.technopolisgroup.com/resources/downloads/661_report_final.pdf>Acesso em: 29 set. 2015.

Rennings, K. (1998). Towards a Theory and Policy of Eco-Innovation - Neoclassical and (Co-) Evolutionary Perspectives. Discussion Paper $n^{\circ}$ 98-24. Mannheim, Centre for European Economic Research (ZEW), 1998. Disponível em: Acesso em: 15 out. 2015. 